\title{
Demonstration of acceleration of relativistic electrons at a dielectric microstructure using femtosecond laser pulses
}

\author{
Kent P. Wootton ${ }^{1, *}$, Ziran Wu ${ }^{1}$, Benjamin M. Cowan ${ }^{2}$, Adi Hanuka ${ }^{1,3}$, Igor V. Makasyuk ${ }^{1}$, \\ Edgar A. Peralta ${ }^{4}$, Ken Soong ${ }^{4}$, Robert L. Byer ${ }^{4}$, and R. Joel England ${ }^{1}$ \\ ${ }^{1}$ SLAC National Accelerator Laboratory, 2575 Sand Hill Road, Menlo Park, California 94025, USA \\ ${ }^{2}$ Tech-X Corporation, 5621 Arapahoe Avenue, Suite A, Boulder, Colorado 80303, USA \\ ${ }^{3}$ Technion-Israel Institute of Technology, Haifa 32000, Israel \\ ${ }^{4}$ Stanford University, 450 Serra Mall, Stanford, California 94305, USA \\ *Corresponding author: wootton@slac.stanford.edu
}

Submitted 8 March 2016; revised 12 May 2016; accepted 16 May 2016.

Acceleration of electrons using laser-driven dielectric microstructures is a promising technology for the miniaturization of particle accelerators. Achieving the desired $\mathrm{GV} \mathrm{m}^{-1}$ accelerating gradients is possible only with laser pulse durations shorter than $\sim 1$ ps. In this Letter, we present the first demonstration of acceleration of relativistic electrons at a dielectric microstructure driven by femtosecond duration laser pulses. Using this technique, an electron accelerating gradient of $690 \pm 100 \mathbf{M V ~ m}^{-1}$ was measured - a record for dielectric laser accelerators. (๑) 2016 Optical Society of America. One print or electronic copy may be made for personal use only. Systematic reproduction and distribution, duplication of any material in this paper for a fee or for commercial purposes, or modifications of the content of this paper are prohibited.

OCIS codes: (230.3990) Micro-optical devices; (140.3295) Laser beam characterization; (320.0320) Ultrafast optics; (050.2770) Gratings.

http://dx.doi.org/10.1364/OL.41.002696

Increasing the accelerating gradient of electron linear accelerators (linacs) by several orders of magnitude would revolutionize their use by dramatically reducing their size. Present compact radio-frequency (rf) free-electron lasers (FELs) operate with an accelerating gradient of $35 \mathrm{MV} \mathrm{m}^{-1}$ over a site length of $\sim 1 \mathrm{~km}[1]$. Desired future FELs of table-top dimensions $(\sim 10 \mathrm{~m})$ require accelerating gradients orders of magnitude higher [2]. One promising approach is to pair a dielectric laser-driven accelerator (DLA) with an optical fiber laser as a scalable electromagnetic power source [3].

Recently, acceleration of relativistic electrons using dielectric grating microstructures has been observed with accelerating gradients of several hundred $\mathrm{MV} \mathrm{m}^{-1}$ [4]. However, simulations indicate that these microstructures should be capable of several $\mathrm{GV} \mathrm{m}^{-1}$ accelerating gradients [5-10]. Motivated by observations of a plateau in damage fluence of dielectric materials for laser pulse durations below $\sim 1$ ps [11], achieving these high gradients without damaging the structure is enabled through the use of short (femtosecond) laser pulses. This has been demonstrated recently with very-low energy electrons [12-14]. In this Letter, we have demonstrated the technique at relativistic electron energy scales, which is ultimately the energy regime these structures need to operate at for most particle accelerator applications.

A consequence of the Lawson-Woodward theorem is that free charged particles are not accelerated by a free-space electromagnetic wave [15]. This can be mitigated by modulating the laser wavefront phase in the presence of a periodic structure boundary. Over one or more periods of the DLA microstructure, this results in electron acceleration [16]. The dielectric microstructure used in this experiment is a 'phase-reset' grating accelerator structure illustrated in Fig. 1 [6]. Structure dimensions are similar to the geometry used in previous work [4, 17]. The structure period $\Lambda$ is set by the criterion for synchronicity with the accelerating mode given by $\Lambda=\beta \lambda$, where $\lambda$ is the laser wavelength and $\beta=v / c \approx 1$, where $v$ is the electron velocity, and $c$ is the speed of light [18].

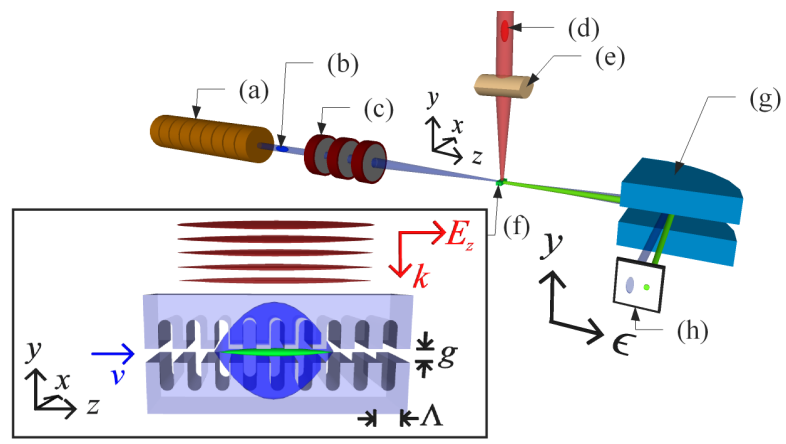

Fig. 1. Schematic of experimental setup. (a) Linear accelerator. (b) Electron beam. (c) Magnetic quadrupole lens. (d) Laser pulse. (e) Cylindrical lens. (f) DLA microstructure with grating period $\Lambda=800 \mathrm{~nm}$, gap $g=400 \mathrm{~nm}$, and length $1 \mathrm{~mm}$. Electrons propagate with velocity $v$ in the $z$-direction. Straggled electrons are indicated in blue [19], and electrons transmitted through the gap are indicated in green. The laser field is incident with wavelength $\lambda=800 \mathrm{~nm}$, wavevector $\vec{k}$, and linear polarization $E_{z}$. (g) Magnetic spectrometer. (h) Detector, indicating straggled and transmitted electron beam populations separated in electron beam energy $\epsilon$. 
Fused silica $\left(\mathrm{SiO}_{2}\right)$ was selected as the substrate material, owing to its high laser damage threshold fluence of $2.0 \mathrm{~J} \mathrm{~cm}^{-2}$ at a laser wavelength of $\lambda=800 \mathrm{~nm}$ under vacuum [20]. For a laser pulse length of $\tau=90 \mathrm{fs}$, this fluence corresponds to an incident electric field of $E_{\mathrm{inc}}=13 \mathrm{GV} \mathrm{m}^{-1}$. The pillar heights of this non-resonant structure are dimensioned to modulate the phase of a linearly polarized laser field by $\pi$. Given the index of refraction of amorphous fused silica is $n=1.5$ at the laser wavelength of $\lambda=800 \mathrm{~nm}$ [21], the pillar height is optimized at $900 \mathrm{~nm}$, however the lithography process used limited the fabricated pillar height to $701 \pm 10 \mathrm{~nm}$, which was used with a $12 \%$ reduction in accelerating gradient [22]. The dielectric accelerator used has an electron beam gap of $g=400 \mathrm{~nm}$.

The laser used was a Ti:sapphire fs regenerative amplifier, seeded by a mode-locked laser oscillator centered at $\lambda=$ $800 \mathrm{~nm}$. The temporal amplitude envelope of the laser pulse (Fig. 2) was measured using the frequency-resolved optical gating (FROG) technique [23]. The measured duration of the laser pulse intensity envelope was $\tau_{i}=64_{-7}^{+11} \mathrm{fs}$ full-width at halfmaximum (FWHM), corresponding to an electric field amplitude envelope of $\tau=90_{-9}^{+16}$ fs FWHM duration. Integration over the temporal envelope of Fig. 2 with a peak accelerating gradient of $G=1 \mathrm{GV} \mathrm{m}^{-1}$ results in a maximum energy gain over any optical phase of $\Delta \epsilon_{1}=35 \pm 5 \mathrm{keV}$.

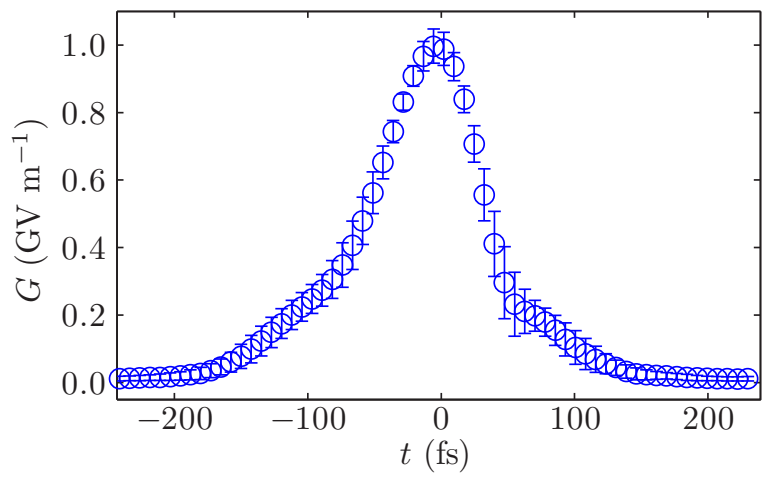

Fig. 2. Measured temporal amplitude envelope of the laser pulse, scaled to a peak field of $1 \mathrm{GV} \mathrm{m}^{-1}$. This was measured using the FROG technique [23, 24], with uncertainties determined using the bootstrap statistical method [25].

As illustrated in Fig. 1, the structure was illuminated asymmetrically from one side with a single laser beam. The laser wavevector $\vec{k}$ was aligned perpendicular to the microstructure by back-propagating the reflection off the top surface of the DLA. The Gaussian beam radii of the laser beam intensity $\left(1 / e^{2}\right)$ at the DLA were measured using a CMOS camera as $w_{\mathrm{lx}}=101 \pm 5 \mu \mathrm{m}, w_{\mathrm{lz}}=480 \pm 10 \mu \mathrm{m}$, corresponding to an rms electric field distribution of $\sigma_{\mathrm{lx}}=71 \pm 4 \mu \mathrm{m}, \sigma_{\mathrm{lz}}=339 \pm 7 \mu \mathrm{m}$. The laser pulse energy was measured as $U=128 \pm 13 \mu \mathrm{J}$. This corresponds to a peak laser fluence of $F=0.17 \pm 0.02 \mathrm{~J} \mathrm{~cm}^{-2}$, or electric field of $E_{\mathrm{inc}}=3.8_{-0.7}^{+0.4} \mathrm{GV} \mathrm{m}^{-1}$.

A beam of electrons was accelerated to energy $\epsilon_{0}=60 \mathrm{MeV}$ by a linear accelerator [26]. Using high-gradient permanent magnet quadrupoles, the electron beam was focused to a waist at the DLA structure. The transverse size of the electron beam was measured using knife edge scans in the horizontal and vertical dimensions at the position of the DLA as $\sigma_{\mathrm{ex}}=28 \pm 3 \mu \mathrm{m}$, $\sigma_{\text {ey }}=22 \pm 1.2 \mu \mathrm{m}$.

Downstream of the DLA, the electron beam was dispersed in energy by a point-to-point focusing spectrometer magnet, as depicted in Fig. 1(g). The electron beam energy distribution was observed on a Lanex phosphor screen imaged by an intensified CCD camera. This distribution was composed of a small population of electrons transmitted ballistically through the $g=400 \mathrm{~nm}$ gap of the DLA channel, and electrons straggling the fused silica substrate and grating pillars with a mean energy loss of $300 \mathrm{keV}$ [19]. Figure 3 shows the measured energy modulation of electrons transmitted through the DLA.

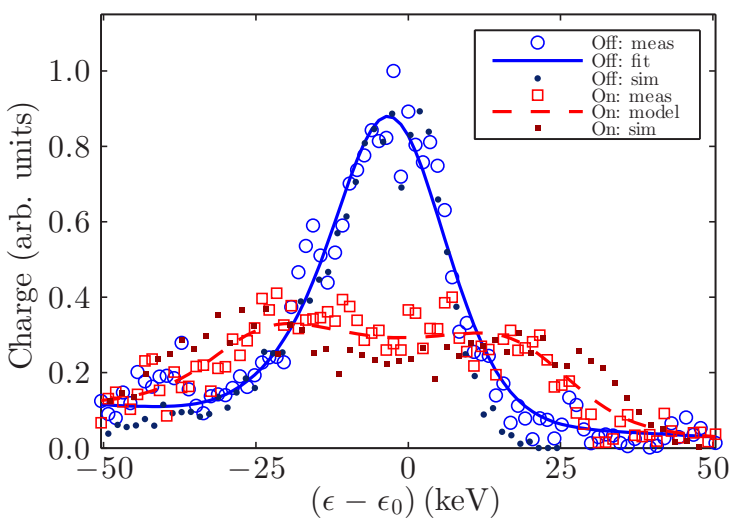

Fig. 3. Measured, fitted and simulated electron beam energy distribution, with the laser off and on. These spectra are a single pair of events corresponding to the highest observed acceleration gradient. With the laser on, electrons are accelerated and decelerated, broadening the energy distribution. Electrons straggled through the fused silica substrate and grating pillars (not shown) were observed with a mean energy loss of $300 \mathrm{keV}$.

The measured electron beam energy spectrum was interpreted using an analytical model of the DLA and particle-incell simulations. In conventional rf accelerators, the electron bunch length is typically much shorter than the wavelength of the accelerating electric field and all electrons in the bunch are accelerated. However since the electron bunch length used in the present experiment ( $\sim 170 \mathrm{fs})$ is much longer than the laser optical cycle $(\lambda / c=2.7 \mathrm{fs})$, electrons experience acceleration or deceleration corresponding to the sinusoidal phase of the incident laser field. Modulation of the electron beam energy spectrum is a result of the injected electron beam phase space, not the DLA microstructure: net acceleration of electrons would be observed if the electron beam were microbunched, for example as demonstrated by an inverse free-electron laser [27].

For the analytical model, the measured laser-off energy spectrum is fitted and subsequently modulated sinusoidally in energy with amplitude $\Delta \epsilon$, and optical phase $\psi$ [4]. This is illustrated in Fig. 4, showing a Lorentzian energy distribution $F\left(\epsilon-\epsilon_{0}\right)$ of an electron bunch significantly longer than the laser optical cycle.

The peak accelerating gradient was determined from the ratio of the measured energy gain $\Delta \epsilon$ to the energy gain from the measured laser pulse temporal distribution, $\Delta \epsilon_{1}$, which would yield a peak accelerating gradient of $1 \mathrm{GV} \mathrm{m}^{-1}$. The half-width at half-maximum (HWHM, $\Gamma / 2)$ of the measured electron beam energy distribution with the laser on in Fig. 3 was $\Gamma / 2=33.8 \pm 1.3 \mathrm{keV}$. From our analytical model, this corresponds to a maximum energy gain of $\Delta \epsilon=24.0 \pm 1.1 \mathrm{keV}$. 


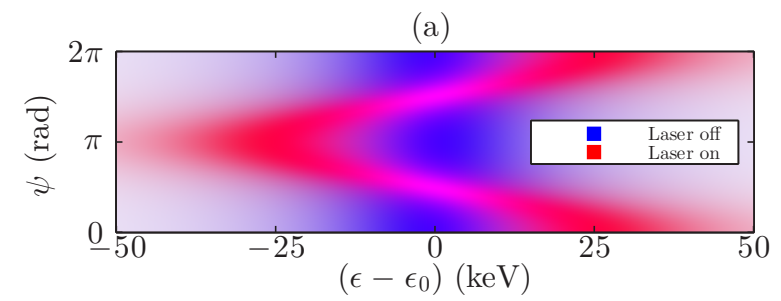

(b)

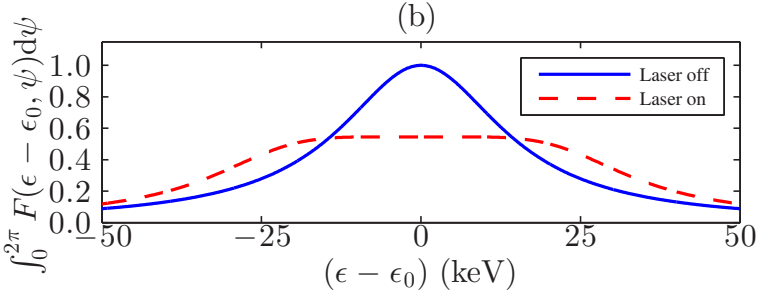

(c)

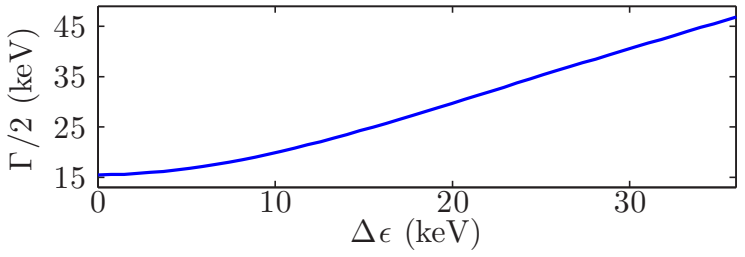

Fig. 4. Analytical model of electron beam energy response to laser-driven microstructure. (a) Electron beam distribution in energy and optical phase, with electron density indicated by color density. For an electron beam longer than a laser optical cycle, the electron beam energy distribution is constant in phase $\psi$ with the laser off, and modulated sinusoidally in phase $\psi$ with the laser on. (b) Integration of (a) over $\psi$, illustrating the change in width of the transmitted population energy distribution with the laser on and off. (c) The half-width at half-maximum (HWHM) $\Gamma / 2$ of the transmitted population as a function of maximum energy gain $\Delta \epsilon$.

For the laser electric field temporal profile illustrated in Fig. 2, this corresponds to an accelerating gradient $G=\left(1 \mathrm{GV} \mathrm{m}^{-1}\right) \times$ $\left(\Delta \epsilon / \Delta \epsilon_{1}\right)=690 \pm 100 \mathrm{MV} \mathrm{m}^{-1}$.

In addition, a complete, self-consistent simulation of the experiment was performed using the high-performance particlein-cell code VSIM using the VORPAL engine $[28,29]$. To account for energy loss and straggling of electrons passing through the fused silica material, two effects were included: bremsstrahlung (using cross-sections tabulated in Ref. [30]) and ionization losses (using the Landau form [31]). Measured values of the laser parameters were used to simulate the laser pulse. Figure 3 shows the simulated beam energy spectra with the laser off and on. The analytical model was fitted to the laseron simulation with an energy gain of $\Delta \epsilon=29.0 \pm 1.0 \mathrm{keV}$, corresponding to a gradient $G=830 \pm 120 \mathrm{MV} \mathrm{m}^{-1}$. Since these time-domain simulations do not include nonlinear material or multi-photon physics, the agreement between simulation and experiment is further confirmation that the microstructure device is operating in the linear regime. The discrepancy between the measured and simulated laser-on accelerating gradients is of the same order as experimental uncertainty, and is consistent with misalignments from ideal geometry $[4,12,13]$.

The measured energy modulation also showed a strong cross correlation between the laser arrival time and electron bunch arrival time, as illustrated in Fig. 5. Using a variable delay stage, the laser pulse arrival time was scanned over a range of several picoseconds about the interaction, observing the energy distribution with the laser off and on. The uncertainty in the measured gradient is comparable to the noise floor of $170 \mathrm{MV} \mathrm{m}^{-1}$ in the arrival time scan. The Gaussian distribution fitted to the laser on distribution has an rms width of $\sigma_{\tau}=2.0 \pm 0.2 \mathrm{ps}$. This agrees within uncertainty with the expected cross correlation rms width of $\sigma_{\mathrm{t}}=1.7 \pm 0.3$ ps resulting from the rms longitudinal laser spot size of $\sigma_{\mathrm{lz}}=339 \pm 7 \mu \mathrm{m}$, laser pulse FWHM duration $\tau=90_{-9}^{+16}$ fs and rms jitter in laser seed synchronization of $480 \mathrm{fs}$ [32].

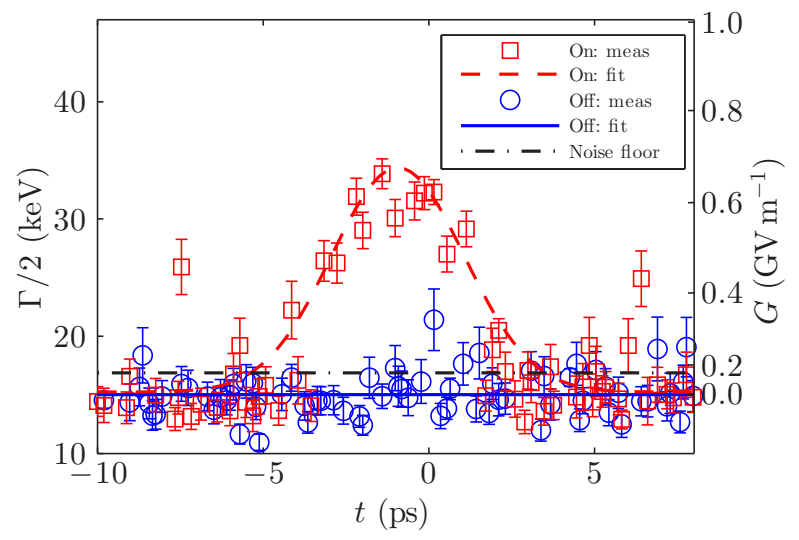

Fig. 5. Measured HWHM $(\Gamma / 2)$ of electron beam energy distribution - the figure of merit for acceleration by the DLA while scanning the laser pulse arrival time. The fitted rms width of the laser on distribution is $\sigma_{\tau}=2.0 \pm 0.2 \mathrm{ps}$. The noise floor is the rms of $\Gamma / 2$ measured for electron beam shots with the laser off.

The major finding of this work was the experimental demonstration that a DLA can be operated in the femtosecond laser pulse regime to provide significant enhancement of accelerating gradient. Using laser pulses compressed to $\tau=90_{-9}^{+16}$ fs duration, the accelerating gradient was significantly increased when compared with a previous experiment using relativistic electrons [4]. The acceleration factor $f=(n G) /\left(\eta E_{\text {inc }}\right)$, where $\eta$ is the ratio of the maximum field in the structure to the incident field, can be used to compare the observed accelerating gradient with simulations $[6,22]$. Given that in this experiment $E_{\mathrm{inc}}=3.8_{-0.7}^{+0.4} \mathrm{GV} \mathrm{m}^{-1}$, for simulations of this structure $\eta=2.6$ [22], the measured value of the acceleration factor $f_{\text {meas }}=0.11 \pm 0.03$ is in agreement with finite-difference timedomain simulations of this DLA structure $f_{\text {sim }}=0.11 \pm 0.02$ [22].

Compared with a previous experiment, the maximum energy gain is reduced, commensurate with the reduction in laser pulse duration [4]. Assuming Gaussian pulse temporal and spatial distributions, in the frame moving with the electron the accelerating gradient is given by [33]:

$$
G=\frac{\Delta \epsilon}{w_{\text {int }} \sqrt{\pi}}, \quad w_{\text {int }}=\left(\frac{1}{w_{\mathrm{lz}}^{2}}+\frac{2 \log _{e} 2}{\left(\beta c \tau_{i}\right)^{2}}\right)^{-1 / 2} .
$$

For parameters of Ref. [4], the interaction length $w_{\text {int }}=280 \mu \mathrm{m}$, while for the present experiment $w_{\text {int }}=16.3 \mu \mathrm{m}$. The principal difference is the laser pulse duration $\tau_{i}$. The reduction in $w_{\text {int }}$ for the present experiment results in a correspondingly lower final energy gain $\Delta \epsilon$. Using pulse-front tilt, operation of a DLA in 
this short-pulse, high-gradient regime could be extended over a greater laser-electron interaction. For relativistic electron beams $(v \approx c)$, a pulse front-tilt of $45^{\circ}$ could be used to match the laser electric field distribution to the electron beam group velocity [6].

The effect of a $1 \%$ wavelength chirp misalignment along the laser pulse is considered. As demonstrated through the radiative inverse process, this DLA has a $14 \mathrm{~nm}$ rms bandpass [20]. Hence the change in instantaneous drive wavelength has a negligible effect on the excitation of the structure. However, chirp introduces a time-dependent phase, such that the field is no longer synchronous with the electron velocity. Consider the electric field represented by:

$$
E(t)=\mathbb{R}\left\{E_{0} \exp \left[(-1+i b)\left(2 \log _{e} 2\right)^{2} t^{2} / \tau^{2}\right]\right\},
$$

where $\tau=90 \mathrm{fs}$, and $\mathbb{R}$ denotes the real component. Integrating over a pulse with peak incident field $E_{0}=690 \mathrm{MV} \mathrm{m}^{-1}$, without chirp $(b=0)$ the maximum acceleration of an electron is $\Delta \epsilon=34.5 \mathrm{keV}$, and for the chirped pulse $(b=2.78)$, $\Delta \epsilon=23.0 \mathrm{keV}$ : the observed energy change is reduced. However, chirped structures may be beneficial to continuous acceleration of non-relativistic electrons, as demonstrated in a recent experiment [34].

The present work has not reached the electric field breakdown limit of the structure fabricated from this material. After the conclusion of the experiment, the DLA was inspected and no damage to the structure was observed. The maximum accelerating gradient observed in the present study was limited by the available laser pulse energy. Future experiments operating with higher laser fluence may achieve higher accelerating gradients.

In conclusion, the present work has demonstrated a DLA microstructure accelerating relativistic electrons with a gradient of $G=690 \pm 100 \mathrm{MV} \mathrm{m}^{-1}$. The present result was achieved using femtosecond scale laser pulses. Future experiments employing pulse-front tilt will extend this high gradient interaction and consequently the total energy gain of the electron beam.

\section{FUNDING.}

U.S. Department of Energy (DE-AC02-76SF00515, DE-FG0213ER41970, DE-SC0008920, DE-AC02-05CH11231); U.S. National Science Foundation (ECS-9731293). Tech-X Corporation.

\section{REFERENCES}

1. T. Inagaki, C. Kondo, H. Maesaka, T. Ohshima, Y. Otake, T. Sakurai, K. Shirasawa, and T. Shintake, Phys. Rev. ST Accel. Beams 17, 080702 (2014).

2. T. Plettner and R. L. Byer, Phys. Rev. ST Accel. Beams 11, 030704 (2008).

3. G. Mourou, B. Brocklesby, T. Tajima, and J. Limpert, Nature Photon. 7, 258 (2013).

4. E. A. Peralta, K. Soong, R. J. England, E. R. Colby, Z. Wu, B. Montazeri, C. McGuinness, J. McNeur, K. J. Leedle, D. Walz, E. B. Sozer, B. Cowan, B. Schwartz, G. Travish, and R. L. Byer, Nature (London) 503, 91 (2013).

5. J. Rosenzweig, A. Murokh, and C. Pellegrini, Phys. Rev. Lett. 74, 2467 (1995).

6. T. Plettner, P. P. Lu, and R. L. Byer, Phys. Rev. ST Accel. Beams 9 , 111301 (2006).

7. B. Naranjo, A. Valloni, S. Putterman, and J. B. Rosenzweig, Phys. Rev. Lett. 109, 164803 (2012)
8. Z. Wu, R. J. England, R. J. Noble, E. A. Peralta, K. Soong, and M. Qi, "Silica rod array for laser driven particle acceleration," in "Proceedings of the North American Particle Accelerator Conference," (JACoW, Geneva, Switzerland, 2013), pp. 141-143.

9. A. Aimidula, C. P. Welsch, G. Xia, K. Koyama, M. Uesaka, M. Yoshida, O. Mete, and Y. Matsumura, Nucl. Instrum. Methods Phys. Res., Sect. A 740, 108 (2014).

10. C.-M. Chang and O. Solgaard, Appl. Phys. Lett. 104, 184102 (2014).

11. A.-C. Tien, S. Backus, H. Kapteyn, M. Murnane, and G. Mourou, Phys. Rev. Lett. 82, 3883 (1999).

12. J. Breuer and P. Hommelhoff, Phys. Rev. Lett. 111, 134803 (2013).

13. K. J. Leedle, R. F. Pease, R. L. Byer, and J. S. Harris, Optica 2, 158 (2015).

14. K. J. Leedle, A. Ceballos, H. Deng, O. Solgaard, R. F. Pease, R. L. Byer, and J. S. Harris, Opt. Lett. 40, 4344 (2015).

15. J. Lawson, IEEE Trans. Nucl. Sci. 26, 4217 (1979).

16. R. J. England, R. J. Noble, K. Bane, D. H. Dowell, C.-K. Ng, J. E. Spencer, S. Tantawi, Z. Wu, R. L. Byer, E. Peralta, K. Soong, C. Chang, B. Montazeri, S. J. Wolf, B. Cowan, J. Dawson, W. Gai, P. Hommelhoff, Y. Huang, C. Jing, C. McGuinness, R. B. Palmer, B. Naranjo, J. Rosenzweig, G. Travish, A. Mizrahi, L. Schachter, C. Sears, G. R. Werner, and R. B. Yoder, Rev. Mod. Phys. 86, 1337 (2014).

17. E. A. Peralta, E. Colby, R. J. England, C. McGuinness, B. Montazeri, K. Soong, Z. Wu, and R. L. Byer, AIP Conf. Proc. 1507, 169 (2012).

18. T. Plettner, R. L. Byer, and B. Montazeri, J. Mod. Opt. 58, 1518 (2011).

19. C. Warner and F. Rohrlich, Phys. Rev. 93, 406 (1954).

20. K. Soong, "Particle accelerator on a wafer: demonstration of electron acceleration and diagnostics with microstructures," Ph.D. thesis, Stanford University (2014).

21. R. Kitamura, L. Pilon, and M. Jonasz, Appl. Opt. 46, 8118 (2007).

22. E. A. Peralta, "Accelerator on a chip: Design, fabrication, and demonstration of grating-based dielectric microstructures for laser-driven acceleration of electrons," Ph.D. thesis, Stanford University (2015).

23. S. Akturk, M. Kimmel, P. O'Shea, and R. Trebino, Opt. Express 11, 491 (2003).

24. D. J. Kane and R. Trebino, Opt. Lett. 18, 823 (1993).

25. Z. Wang, E. Zeek, R. Trebino, and P. Kvam, J. Opt. Soc. Am. B 20, 2400 (2003).

26. E. Colby, R. Noble, D. Palmer, R. Siemann, and J. Spencer, "Beam dynamics studies for a laser acceleration experiment," in "Proceedings of the $21^{\text {st }}$ Particle Accelerator Conference," (IEEE, Piscataway, NJ, USA, 2005), pp. 2024-2026.

27. C. M. S. Sears, E. Colby, R. J. England, R. Ischebeck, C. McGuinness, J. Nelson, R. Noble, R. H. Siemann, J. Spencer, D. Walz, T. Plettner, and R. L. Byer, Phys. Rev. ST Accel. Beams 11, 101301 (2008).

28. C. Nieter and J. R. Cary, J. Comput. Phys. 196, 448 (2004).

29. Tech-X Corporation, "VSim," https://www.txcorp.com/vsim.

30. S. M. Seltzer and M. J. Berger, At. Data and Nucl. Data Tables 35, 345 (1986).

31. L. D. Landau, J. Phys. (Moscow) 8, 201 (1944).

32. D. T. Palmer and R. Akre, "Experimental measurements of the ORION photoinjector drive laser oscillator subsystem," in "Proceedings of the 2003 Particle Accelerator Conference," (IEEE, Piscataway, NJ, USA, 2003), pp. 2107-2109.

33. J. Breuer, R. Graf, A. Apolonski, and P. Hommelhoff, Phys. Rev. ST Accel. Beams 17, 021301 (2014).

34. J. McNeur, M. Kozák, N. Schönenberger, K. J. Leedle, H. Deng, A. Ceballos, H. Hoogland, A. Ruehl, I. Hartl, O. Solgaard, J. S. Harris, R. L. Byer, and P. Hommelhoff, "Elements of a dielectric laser accelerator," (2016). ArXiv:1604.07684 [physics.acc-ph]. 


\section{REFERENCES}

1. T. Inagaki, C. Kondo, H. Maesaka, T. Ohshima, Y. Otake, T. Sakurai, K. Shirasawa, and T. Shintake, "High-gradient c-band linac for a compact x-ray free-electron laser facility," Phys. Rev. ST Accel. Beams 17, 080702 (2014).

2. T. Plettner and R. L. Byer, "Proposed dielectric-based microstructure laser-driven undulator," Phys. Rev. ST Accel. Beams 11, 030704 (2008).

3. G. Mourou, B. Brocklesby, T. Tajima, and J. Limpert, "The future is fibre accelerators," Nature Photon. 7, 258-261 (2013).

4. E. A. Peralta, K. Soong, R. J. England, E. R. Colby, Z. Wu, B. Montazeri, C. McGuinness, J. McNeur, K. J. Leedle, D. Walz, E. B. Sozer, B. Cowan, B. Schwartz, G. Travish, and R. L. Byer, "Demonstration of electron acceleration in a laser-driven dielectric microstructure," $\mathrm{Na}$ ture (London) 503, 91-94 (2013).

5. J. Rosenzweig, A. Murokh, and C. Pellegrini, "A proposed dielectricloaded resonant laser accelerator," Phys. Rev. Lett. 74, 2467-2470 (1995).

6. T. Plettner, P. P. Lu, and R. L. Byer, "Proposed few-optical cycle laserdriven particle accelerator structure," Phys. Rev. ST Accel. Beams 9, 111301 (2006).

7. B. Naranjo, A. Valloni, S. Putterman, and J. B. Rosenzweig, "Stable charged-particle acceleration and focusing in a laser accelerator using spatial harmonics," Phys. Rev. Lett. 109, 164803 (2012).

8. Z. Wu, R. J. England, R. J. Noble, E. A. Peralta, K. Soong, and M. Qi, "Silica rod array for laser driven particle acceleration," in "Proceedings of the North American Particle Accelerator Conference," (JACoW, Geneva, Switzerland, 2013), pp. 141-143.

9. A. Aimidula, C. P. Welsch, G. Xia, K. Koyama, M. Uesaka, M. Yoshida, O. Mete, and Y. Matsumura, "Numerical investigations into a fiber laser based dielectric reverse dual-grating accelerator," Nucl. Instrum. Methods Phys. Res., Sect. A 740, 108 - 113 (2014).

10. C.-M. Chang and O. Solgaard, "Silicon buried gratings for dielectric laser electron accelerators," Appl. Phys. Lett. 104, 184102 (2014).

11. A.-C. Tien, S. Backus, H. Kapteyn, M. Murnane, and G. Mourou, "Short-pulse laser damage in transparent materials as a function of pulse duration," Phys. Rev. Lett. 82, 3883-3886 (1999).

12. J. Breuer and P. Hommelhoff, "Laser-based acceleration of nonrelativistic electrons at a dielectric structure," Phys. Rev. Lett. 111, 134803 (2013).

13. K. J. Leedle, R. F. Pease, R. L. Byer, and J. S. Harris, "Laser acceleration and deflection of $96.3 \mathrm{keV}$ electrons with a silicon dielectric structure," Optica 2, 158-161 (2015).

14. K. J. Leedle, A. Ceballos, H. Deng, O. Solgaard, R. F. Pease, R. L. Byer, and J. S. Harris, "Dielectric laser acceleration of sub-100 keV electrons with silicon dual-pillar grating structures," Opt. Lett. 40, 4344-4347 (2015).

15. J. Lawson, "Lasers and accelerators," IEEE Trans. Nucl. Sci. 26, 42174219 (1979).

16. R. J. England, R. J. Noble, K. Bane, D. H. Dowell, C.-K. Ng, J. E. Spencer, S. Tantawi, Z. Wu, R. L. Byer, E. Peralta, K. Soong, C. Chang, B. Montazeri, S. J. Wolf, B. Cowan, J. Dawson, W. Gai, P. Hommelhoff, Y. Huang, C. Jing, C. McGuinness, R. B. Palmer, B. Naranjo, J. Rosenzweig, G. Travish, A. Mizrahi, L. Schachter, C. Sears, G. R. Werner, and R. B. Yoder, "Dielectric laser accelerators," Rev. Mod. Phys. 86, 1337-1389 (2014).

17. E. A. Peralta, E. Colby, R. J. England, C. McGuinness, B. Montazeri, K. Soong, Z. Wu, and R. L. Byer, "Design, fabrication, and testing of a fused-silica dual-layer grating structure for direct laser acceleration of electrons," AIP Conf. Proc. 1507, 169-177 (2012).

18. T. Plettner, R. L. Byer, and B. Montazeri, "Electromagnetic forces in the vacuum region of laser-driven layered grating structures," J. Mod. Opt. 58, 1518-1528 (2011).

19. C. Warner and F. Rohrlich, "Energy loss and straggling of electrons," Phys. Rev. 93, 406-407 (1954).

20. K. Soong, "Particle accelerator on a wafer: demonstration of electron acceleration and diagnostics with microstructures," Ph.D. thesis, Stanford University (2014).
21. R. Kitamura, L. Pilon, and M. Jonasz, "Optical constants of silica glass from extreme ultraviolet to far infrared at near room temperature," Appl. Opt. 46, 8118-8133 (2007).

22. E. A. Peralta, "Accelerator on a chip: Design, fabrication, and demonstration of grating-based dielectric microstructures for laser-driven acceleration of electrons," Ph.D. thesis, Stanford University (2015).

23. S. Akturk, M. Kimmel, P. O'Shea, and R. Trebino, "Measuring pulsefront tilt in ultrashort pulses using GRENOUILLE," Opt. Express 11, 491-501 (2003).

24. D. J. Kane and R. Trebino, "Single-shot measurement of the intensity and phase of an arbitrary ultrashort pulse by using frequency-resolved optical gating," Opt. Lett. 18, 823-825 (1993).

25. Z. Wang, E. Zeek, R. Trebino, and P. Kvam, "Determining error bars in measurements of ultrashort laser pulses," J. Opt. Soc. Am. B 20, 2400-2405 (2003)

26. E. Colby, R. Noble, D. Palmer, R. Siemann, and J. Spencer, "Beam dynamics studies for a laser acceleration experiment," in "Proceedings of the $21^{\text {st }}$ Particle Accelerator Conference," (IEEE, Piscataway, NJ, USA, 2005), pp. 2024-2026.

27. C. M. S. Sears, E. Colby, R. J. England, R. Ischebeck, C. McGuinness, J. Nelson, R. Noble, R. H. Siemann, J. Spencer, D. Walz, T. Plettner and R. L. Byer, "Phase stable net acceleration of electrons from a twostage optical accelerator," Phys. Rev. ST Accel. Beams 11, 101301 (2008).

28. C. Nieter and J. R. Cary, "VORPAL: a versatile plasma simulation code," J. Comput. Phys. 196, 448 - 473 (2004).

29. Tech-X Corporation, "VSim," https://www.txcorp.com/vsim.

30. S. M. Seltzer and M. J. Berger, "Bremsstrahlung energy spectra from electrons with kinetic energy $1 \mathrm{kev}-10 \mathrm{gev}$ incident on screened nuclei and orbital electrons of neutral atoms with $z=1-100$," At. Data and Nucl. Data Tables 35, $345-418$ (1986).

31. L. D. Landau, "On the energy loss of fast particles by ionization," J. Phys. (Moscow) 8, 201-205 (1944).

32. D. T. Palmer and R. Akre, "Experimental measurements of the ORION photoinjector drive laser oscillator subsystem," in "Proceedings of the 2003 Particle Accelerator Conference," (IEEE, Piscataway, NJ, USA, 2003), pp. 2107-2109.

33. J. Breuer, R. Graf, A. Apolonski, and P. Hommelhoff, "Dielectric laser acceleration of nonrelativistic electrons at a single fused silica grating structure: Experimental part," Phys. Rev. ST Accel. Beams 17, 021301 (2014).

34. J. McNeur, M. Kozák, N. Schönenberger, K. J. Leedle, H. Deng, A. Ceballos, H. Hoogland, A. Ruehl, I. Hartl, O. Solgaard, J. S. Harris, R. L. Byer, and P. Hommelhoff, "Elements of a dielectric laser accelerator," (2016). ArXiv:1604.07684 [physics.acc-ph]. 